\title{
How China's “Double-Eleven” Day Challenges Confucianism: An Abstract
}

\author{
Wenkai Zhou, Yu-Feng L. Lee, and Michael R. Hyman
}

\begin{abstract}
Double-Eleven" Day (aka 11.11 [November 11th], Singles Day, and Bachelors Day) is an e-commerce shopping spree celebrated by millions of Chinese e-shoppers. Since 2013, Chinese e-tailers have experienced record 1-day sales on that date. Qualitative secondary data about 'Double-Eleven' Day e-shoppers suggests they are short-term oriented and individualistic, anti-hierarchical in buyerseller relationships, focused on immediate consumption and hedonic pleasure, and prone to spontaneous trust and superficial interactions when online. In contrast, traditional Chinese shoppers conform to long-established Chinese personal selling strategies by being long-term oriented, collectivistic, and seekers of long-term mutual trust in hierarchical buyer-seller relationships. Hence, Chinese e-tailers should adapt their selling practices to Chinese e-shoppers' attitudes and behaviors.
\end{abstract}

References Available Upon Request.

W. Zhou $(\bowtie) \bullet$ Y.-F. L. Lee • M.R. Hyman

New Mexico State University, Las Cruces, NM, USA

e-mail:wkzhou@nmsu.edu; wlin@nmsu.edu; mhyman@nmsu.edu 